\title{
ИССЛЕДОВАНИЕ РЕЖИМОВ УХУДШЕННОГО ТЕПЛООБМЕНА ПРИ ТЕЧЕНИИ ВОДЫ СВЕРХКРИТИЧЕСКИХ ПАРАМЕТРОВ В ВЕРТИКАЛЬНОЙ ТРУБЕ
}

\author{
Авраменко А.А., член-корреспондент НАН Украины, Ковецкая М.М., к.т.н., \\ Кондратьева Е.А., к.т.н., Тыринов А.И., к.т.н. \\ Институт технической теплофизики НАН Украинь, ул. Желябова 2а, Киев 03680, Украина
}

Представлено результати чисельного моделювання теплообмінних процесів плину води надкритичного тиску в вертикальній трубі, що обігрівається. Досліджені умови виникнення режимів погіршеного теплообміну.
Представлены результаты численного моделирования теплообменных процессов при течении воды сверхкритического давления в вертикальной обогреваемой трубе. Исследованы условия возникновения режимов ухудшенного теплообмена.
The results of numeral simulations of heat transfer processes are presented for the flow of supercritical water in the vertical heated pipe. The conditions of the worsened heat transfer are investigated.

Библ.10, рис. 5.

Ключевые слова: численное моделирование, вертикальная труба, сверхкритическое давление.

$G_{k}$ - генерация турбулентной энергии;

$g$ - ускорение свободного падения;

$h$ - энтальпия;

$k$ - кинетическая энергия турбулентности;

$\operatorname{Pr}$ - число Прандтля;

$p$ - давление;

$q$ - плотность теплового потока;

$S$ - тензор скоростей деформации;

$s-$ постоянная, равная 0,97 ;

$T$ - температура;

$V$ - вектор скорости теплоносителя;

$w$ - скорость;

$y$ - поперечная координата;

$z$ - продольная координата;

\section{Введение}

Опыт эксплуатации энергоблоков ТЭС с водой сверхкритических параметров показал наличие проблем связанных с возникновением в теплообменных трубах режимов с ухудшенным теплообменом. Для обоснования надежной работы энергоблоков ТЭС было проведено большое количество теоретических и экспериментальных исследований теплообмена при течении воды сверхкритических параметров в трубах. Исследования показали наличие режимов с нормальным теплообменом, характеризующимся монотонным изменением температуры стенки по $\delta_{n m}-$ тензор Кронекера второго ранга;

$\varepsilon-$ скорость диссипации;

$\overrightarrow{\mathrm{k}}-$ opт;

$\mu-$ вязкость;

$\rho-$ плотность.

Индексы:

вх - входной параметр;

ж-жидкость;

eff - эффективный;

$k$ - кинетический;

$t$ - турбулентный;

$\varepsilon-$ относиться к скорости диссипации.

длине обогреваемой трубы и с ухудшенным теплообменом, характеризующимся резко выраженным локальным повышением температуры стенки. Попытки описать достаточно точно теплоотдачу в режимах ухудшенного теплообмена не всегда приводят к желаемому результату.

Исследованию теплообмена при сверхкритическом давлении посвящено много работ [1-4]. Отмечается, что сложность описания процессов теплообмена при течении жидкости сверхкритического давления требует изучения структуры потока. В работе [5] проанализированы экспериментальные данные по теплообмену при 
сверхкритическом давлении в трубах и пучках стержней. В результате сильного уменьшения плотности среды при приближении к критической температуре возникает термическое ускорение потока, что приводит к снижению турбулентности. При подъемном течении теплоносителя в вертикальной трубе профиль скорости приобретает М-образную форму с максимумом вблизи стенки. В этой области образуется запирающий для переноса теплоты слой, что приводит к ухудшению теплообмена и повышению температуры стенки. Нет единого мнения по определению граничного значения теплового потока, превышение которого приводит к возникновению режимов с ухудшенной теплоотдачей и его зависимости от режимных и геометрических параметров. На основании экспериментальных данных [6] можно сделать вывод о том, что при течении воды сверхкритических параметров в трубах нижняя граница возникновения зоны с ухудшенным теплообменом определяется условием $q_{\mathrm{c}} / \rho w \geq 0,6$ кДж/кг. Особенно большой разброс данных по определению границы ухудшенного теплообмена возникает при малых массовых скоростях теплоносителя.

Для определения границ области ухудшенного теплообмена в каналах используют теоретические методы решения задачи конвективного теплообмена при течении жидкости с переменными теплофизическими свойствами. Актуальным остается вопрос повышения точности расчета течения и теплообмена в околокритической области параметров теплоносителя в связи с переходом на новый стандарт свойств воды IF-97. В работе представлены результаты численного моделирования подъемного течения воды в вертикальной обогреваемой трубе диаметром 16 мм, длиной 4 м при давлении 24,5 МПа, массовой скорости $250 \mathrm{\kappa г} /\left(\mathrm{M}^{2} \mathrm{c}\right)$ для температуры на входе $T_{\text {вх }}=200,230,250{ }^{\circ} \mathrm{C}$. На стенке трубы задавался постоянный тепловой поток.

Численное моделирование течения воды в трубе выполнено на основе RNG $k$ - $\varepsilon$ модели турбулентности, включающей систему уравнений сохранения массы, количества движения, энергии теплоносителя, а также уравнения для кинетической энергии турбулентности и скорости диссипации энергии [7]. Теплофизические свой- ства воды сверхкритических параметров описывались согласно формуляции IF-97 [8]. Расчеты проводились методом контрольного объема [9].

Основные уравнения математической модели:

$$
\begin{aligned}
& \rho[\frac{\partial V}{\partial t}+\underbrace{s \frac{\partial \nabla\left(\mu_{t} / \rho\right)}{\partial t}}_{\mathrm{I}}+(V \cdot \nabla) V]=-\nabla p+ \\
& +\nabla \cdot\left[\mu_{\mathrm{eff}}\left(\nabla V+(\nabla V)^{T}-\frac{2}{3} \nabla V \delta_{\mathrm{nm}}\right)\right]+F \overrightarrow{\mathrm{k}},
\end{aligned}
$$

$\frac{\partial \rho h}{\partial t}+\underbrace{s \frac{\partial}{\partial t} \nabla \cdot\left(\frac{\left(\mu_{t}\right)}{\sqrt{2} S} \nabla h\right)}_{\mathrm{I}}+\nabla(\rho h V)=\nabla \cdot\left(\frac{\mu_{\text {eff }}}{\operatorname{Pr}_{\text {eff }}} \nabla h\right),(2)$

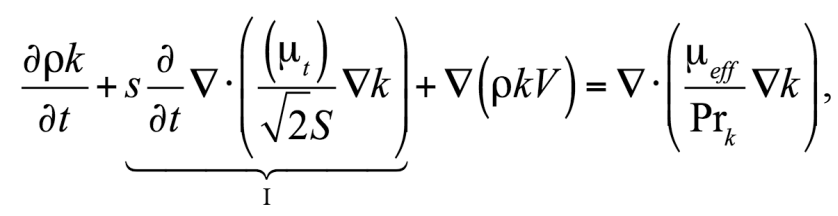

$$
\frac{\partial \rho \varepsilon}{\partial t}+\underbrace{s \frac{\partial}{\partial t} \nabla \cdot\left(\frac{\left(\mu_{t}\right)}{\sqrt{2} S} \nabla \varepsilon\right)}_{\mathrm{I}}+\nabla(\rho \varepsilon V)=\nabla \cdot\left(\frac{\mu_{\text {leff }}}{\operatorname{Pr}_{\varepsilon}} \nabla \varepsilon\right)+S_{\varepsilon},
$$

$G_{k}=\mu\left(\nabla V+(\nabla V)^{T}-\frac{2}{3} \nabla V \delta_{\mathrm{nm}}\right)^{2}$

$S=\frac{1}{2}\left(\frac{\partial u_{i}}{\partial x_{j}}+\frac{\partial u_{j}}{\partial x_{i}}\right), S_{\varepsilon}=\rho \frac{\varepsilon}{k}\left(C_{1} G_{k}-C_{2} \varepsilon\right), C_{1}=1,42$, $C_{2}=1,68$,

где $F=\rho g-$ подъемная сила, $\nabla-$ тензорная дивергенция, $\nabla V$ - тензорный градиент, который преобразует вектор (тензор первого ранга) в тензор второго ранга, $(\nabla V)^{T}$ - сопряженный тензорный градиент, I - дополнительное слагаемое, учитывающее нестационарность турбулентных процессов.

Результаты расчета. На рис. 1, 2 показано распределение температуры стенки по длине трубы при изменении температуры воды на входе и тепловой нагрузки.

Режим ухудшенного теплообмена возникает при значении параметра $q / \rho w=1,2$ кДж/кг и тем- 


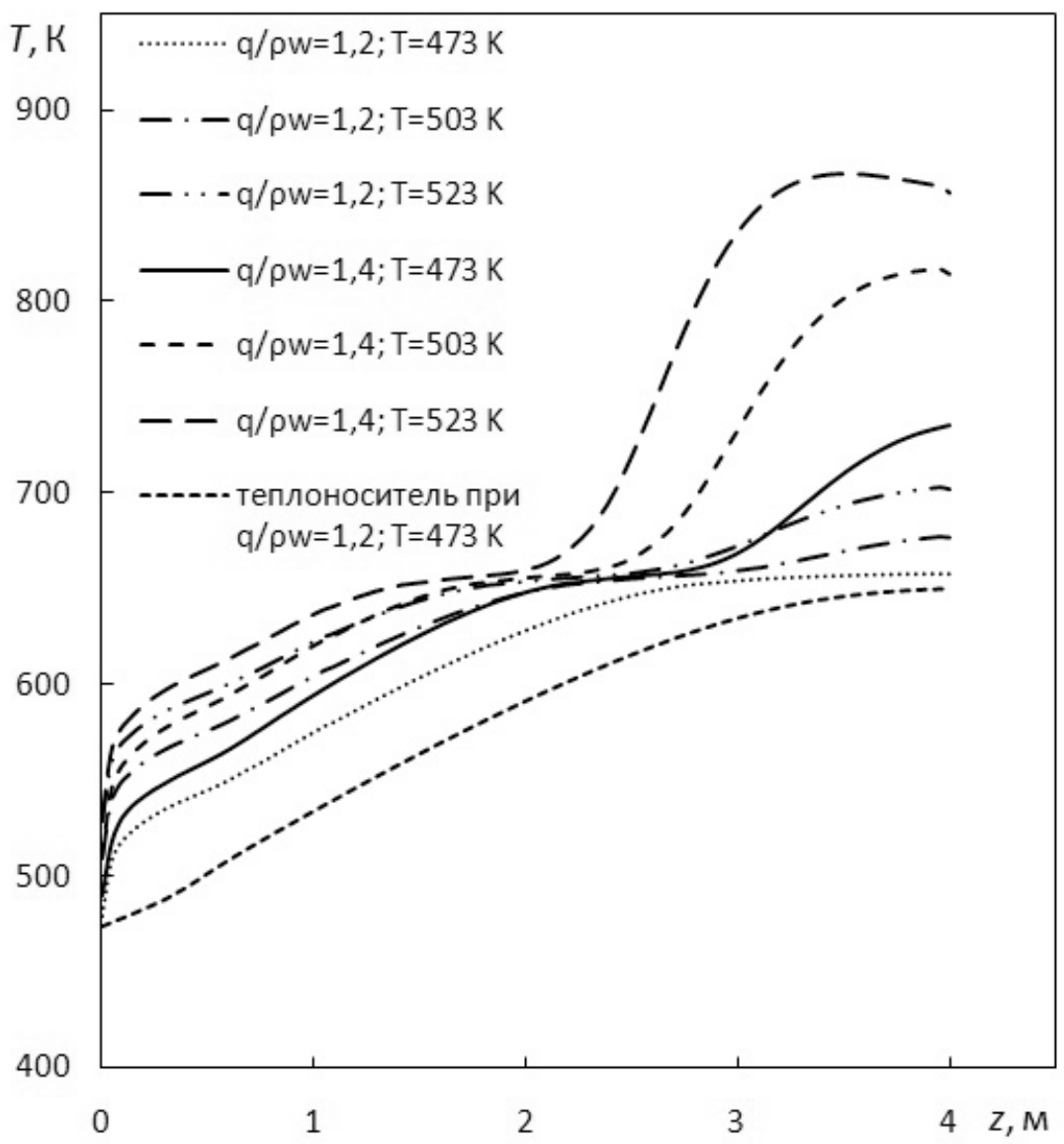

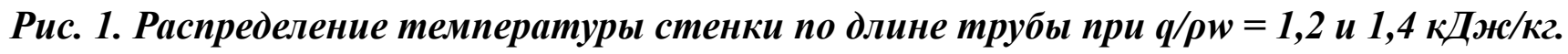

пературе воды на входе $T_{\text {вх }}=250{ }^{\circ} \mathrm{C}$. При меньших значениях температуры на входе наблюдается режим нормального теплообмена с монотонным изменением температуры стенки. Зона ухудшенного теплообмена наблюдается на выходе из трубы. С ростом тепловой нагрузки режим ухудшенного теплообмена возникает при меньшей температуре воды на входе. С ростом входной температуры зона ухудшенного теплообмена сдвигается к входу. Для режимов, представленных на рис. 1 с ростом температуры воды на входе максимальное значение температуры стенки растет. В режимах с большей тепловой нагрузкой $q / \rho w=1,6$ кДж/кг с увеличением температуры теплоносителя на входе зона ухудшенного теплообмена тоже сдвигается к входу, однако максимальное значение температуры стенки меняется слабо (рис. 2).

Резкое увеличение температуры стенки наблюдается в области перехода температуры теплоносителя к сверхкритическому значению. В этой области наблюдается резкое ускорение потока и деформация профиля скорости. На рис. 3 представлены профили скорости в разных сечениях по длине трубы в режиме $q / \rho w=1,4$ кДж/кг, $T_{\mathrm{Bx}}=250^{\circ} \mathrm{C}$.

В сечении $z=3,4$ м, где температура стенки достигает максимального значения (рис. 1), профиль скорости имеет М-образную форму с максимумом вблизи стенки, что согласуется с результатами исследований, представленных в работе [10]. В этой работе представлены результаты расчета теплообмена и гидродинамики при течении воды в вертикальной обогреваемой трубе в диапазоне давлений 22,5...29,5 МПа, энтальпии потока 500...3500 кДж/кг, массовой скорости $250 \ldots 3000$ кг/ $\left(\mathrm{M}^{2} \mathrm{c}\right)$ и тепловой нагрузки, соответствующей параметру $q / \rho w=0,2 \ldots 1,2$ кДж/кг. Авторы отмечают, что представленный интегральный метод расчета конвективного теплообмена при течении воды сверхкритического давления позволяет получить достоверные расчет- 


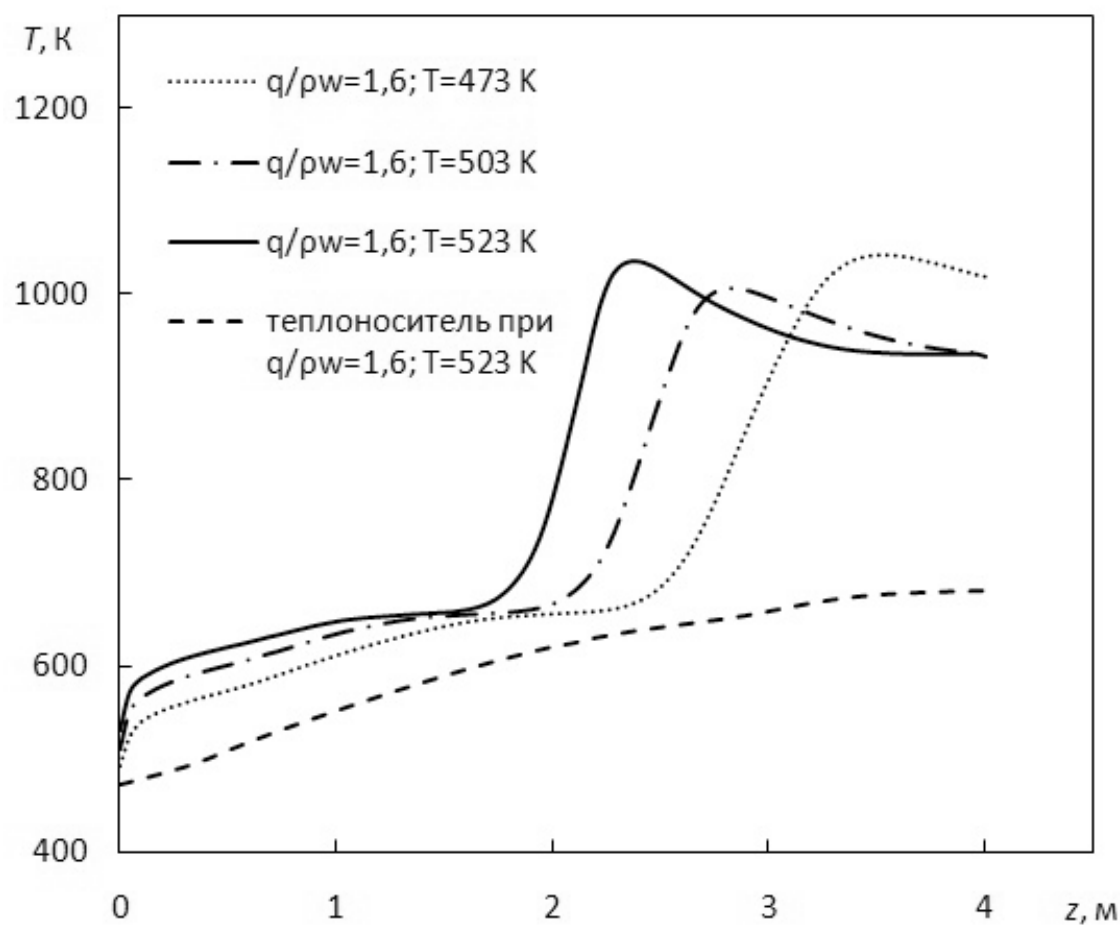

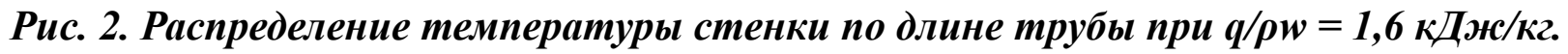

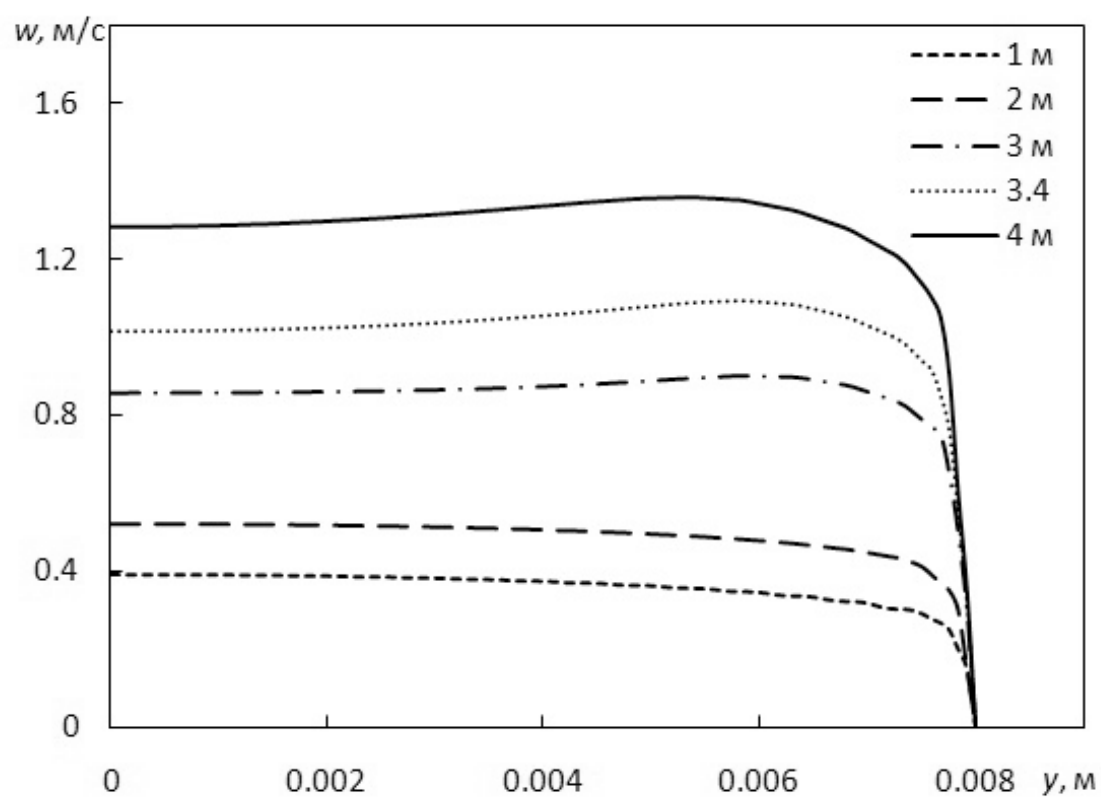

Рис. 3. Распределение скорости теплоносителя в сечениях по длине трубы в режниме $q / \rho w=1,4 \kappa$ Кюс/кг, $T_{\text {вx }}=250{ }^{\circ} \mathrm{C}$.

ные данные только при «умеренных» тепловых нагрузках, соответствующих области нормального теплообмена. Процесс ухудшения теплоотдачи, вызванный перестройкой структуры потока, интегральная методика не описывает. Од- нако результаты расчета, полученные при «больших» тепловых нагрузках, представляют интерес с точки зрения моделирования условий теплообмена при течении с М-образным профилем скорости. На рис. 4 представлено сравнение распре- 


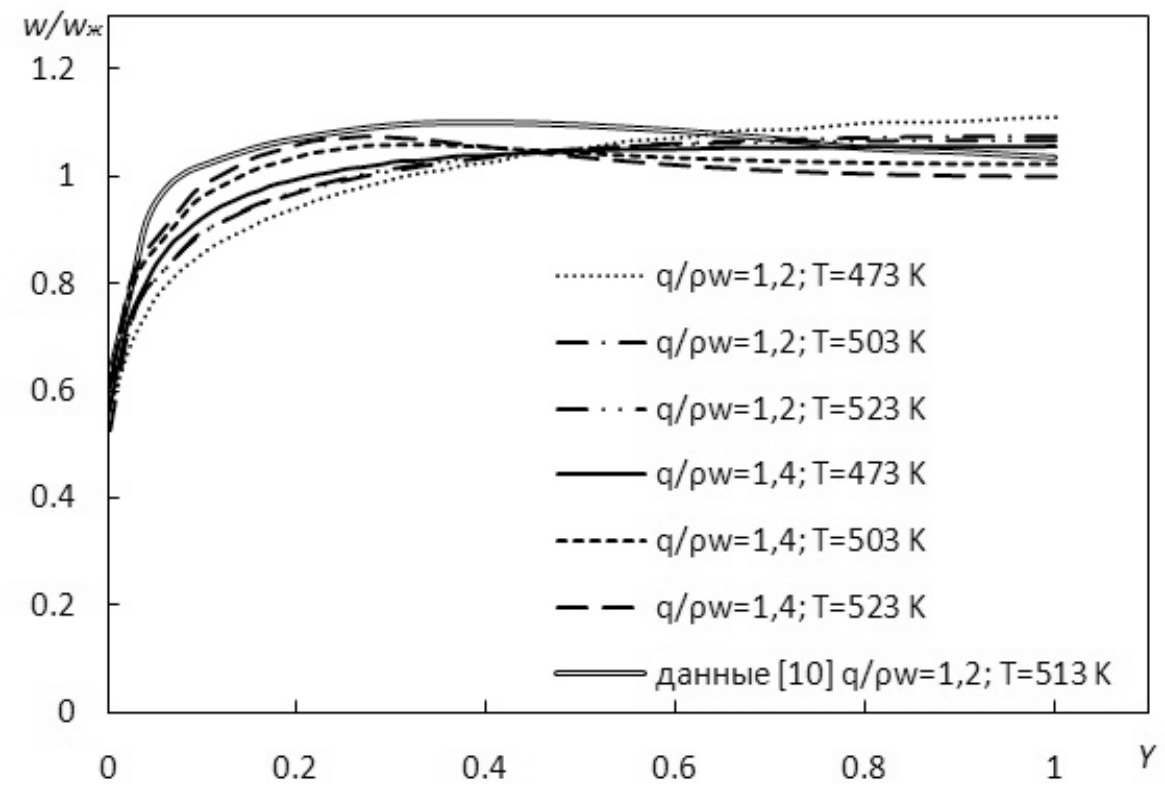

Рис. 4. Распределение относительной скорости теплоносителя в сечениях с максимальным значением температуры стенки.

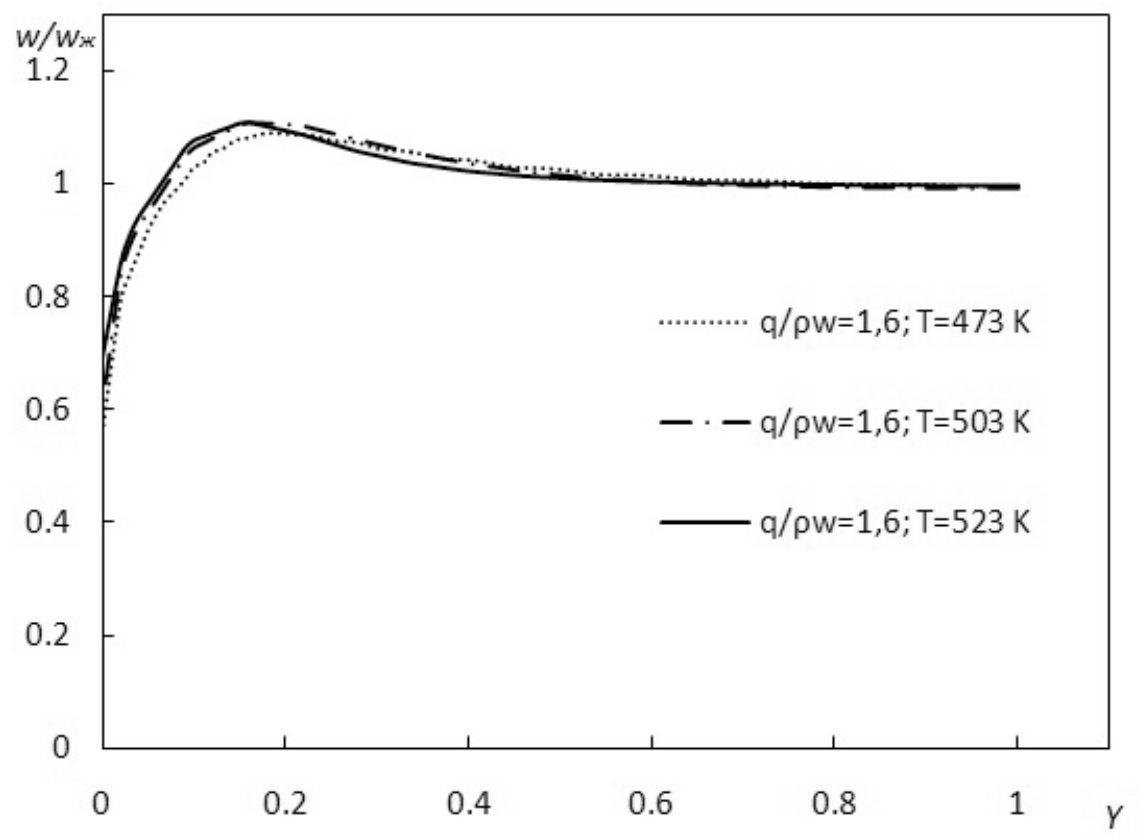

Рис. 5. Распределение относительной скорости теплоносителя в сечениях с максимальным значением температуры стенки.

деления по сечению трубы относительной скорости $w / w_{\text {ж }}$ в сечениях с максимальным значением температуры стенки и результатов, полученных в работе [10] при значении параметра $q / \rho w=1,2$ кДж/кг. Средняя скорость жидкости $w_{\text {ж }}$ определялась путем интегрирования профиля скорости по сечению трубы. Наблюдается качественное согласование результатов, однако, для режимов с большей тепловой нагрузкой $(q / \rho w=$ $=1,4$ кДж/кг).

На рис. 5 представлено распределение по сечению относительной скорости $w / w_{\text {ж }}$ в се- 
чениях с максимальным значением температуры стенки при значении параметра $q / \rho w=$ $=1,6$ кДж/кг для разных значений температуры воды на входе.

Представленные результаты свидетельствуют о том, что с увеличением тепловой нагрузки профиль относительной скорости деформируется сильнее, максимальное значение скорости сдвигается к стенке. В режимах, представленных на рис. 4, наблюдается существенная деформация профиля относительной скорости с изменением температуры воды на входе. Профиль приобретает более выраженную М-образную форму, что согласуется с ростом максимального значения температуры стенки в обасти ухудшенного теплообмена при увеличении температуры воды на входе (рис. 1). В режимах, представленных на рис. 5, профиль относительной скорости меняется незначительно с увеличением температуры воды на входе, соответственно и максимальное значение температуры стенки в зоне ухудшенного теплообмена меняется слабо (рис. 2).

\section{Выводы}

1. Численное моделирование гидродинамики и теплообмена при течении воды сверхкритического давления в вертикальной обогреваемой трубе показало, что режимы ухудшенного теплообмена возникают при значении параметра $q / \rho w \geq 1,2$ кДж/кг.

2. С увеличением температуры воды на входе локальная зона ухудшенного теплообмена сдвигается к входу, максимальное значение температуры стенки увеличивается.

3. С увеличением плотности теплового потока профиль скорости существенно деформируется, приобретает М-образную форму с максимумом вблизи стенки. Возникновение режимов ухудшенного теплообмена связано с изменением структуры потока, существенной деформацией профиля скорости, вызванной термическим ускорением потока вблизи стенки, что существенно снижает отвод теплоты от стенки в ядро потока.

\section{ЛИТЕРАТУРА}

1. Курганов B.A. Теплообмен в трубах при сверхкритических давлениях теплоносителя: некоторые итоги научного исследования// Труды PHKT-4. - 2006. - T.1. - C.74 - 83.

2. Pioro I.L., Khartabil H.F., Duffey R.B. Heat transfer to supercritical fluids flowing in channels-empirical correlations (survey)// Nucl. Eng. Design. - 2004. - v.230. - P. 69 - 91.

3. Cheng $X$, Schulenberg $T$. Heat transfer at supercritical pressure - literature review and application to a HPLWR// Wissenscha ftliche Berichte FZKA6609 / Forschungszentrum, Karlsruhe, May, 2001.

4. Pioro I., Khartabil H., Duffey R. Heat transfer at supercritical pressures (survey)// Proc. 11-th Intern. Conf. on Nucl. Eng. Tokyo, Japan, April 20 - 23. - 2003.Paper ICONE 11-36454.

5. Грабежная В.А., Кириллов П.Л. Теплообмен при сверхкритических давлениях и границы ухудшенного теплообмена// Теплоэнергетика. - 2006. - №4. - С.46- 51.

6. Кириллов П.Л., Ложкин В.В., Смирнов A.M. Исследование границ ухудшенных режимов в каналах при сверхкритических давлениях // Препринт ФЭИ-2988. - 2003.

7. Авраменко А.А., Басок Б.И., Дмитренко Н.П., Ковецкая М.М., Тыринов А.И., Давыденко Б.В. Ренормализационно групповой анализ турбулентности. Киев. ТОВ «ЕКСПРЕС». 2013. - $300 \mathrm{c}$.

8. Александров А.А., Орлов К.А., Очков В.Ф. Теплофизические свойства рабочих веществ теплоэнергетики. М.: Изд. Дом МЭИ. - 2009.

9. Роуч П. Вычислительная гидродинамика. М.: Мир. - 1980. - 616 с.

10. Курганов В.А., Маслакова И.В. Интегральный метод расчета стабилизированной теплоотдачи в трубах в однофазной околокритической области // Теплофизика высоких температур. - 2010. - Т. 48, № 4. - С. 568 - 581 . 


\section{RESEARCH OF MODES OF THE WORSENED HEAT TRANSFER FOR THE FLOW OF SUPERCRITICAL PARAMETERS IN THE VERTICAL PIPE}

\section{A.A. Avramenko, M.M. Kovetskaya, E.A. Kondratieva, A.I. Tyrinov}

Institute of Engineering Thermophysics of the National Academy of Sciences of Ukraine, 03057, Kiev, vul. Zhelyabova 2a, Ukraine

This paper analyzes the processes of heat transfer for the flow of supercritical water in the vertical heated pipe. Numerical study of the processes was based on the $k$ - $\varepsilon$ RNG turbulence model. The results of simulations allow analyzing character of thermophysical and hydrodynamic processes. The conditions of occurred of worsened heat and the effects of influence of the inlet temperature and the parameter $q / \rho w$ are investigated.

References 10, fig. 5 .

Key words: numerical modeling, vertical pipe, supercritical pressure.

1. Kurganov $V$. Heat transfer in tubes at supercritical pressures of media: some results of science studies // Works of Russian national conference of heat exchange -4 . -2006 . - v.1. P. 74 - 83. (Rus.)

2. Pioro I.L., Khartabil H.F., Duffey R.B. Heat transfer to supercritical fluids flowing in channels-empirical correlations (survey) // Nucl. Eng. Design. - 2004. - v. 230. - P. $69-91$.
3. Cheng $X$, Schulenberg T. Heat transfer at supercritical pressure - literature review and application to a HPLWR // Wissenscha ftliche Berichte FZKA6609 / Forschungszentrum, Karlsruhe, May, 2001.

4. Pioro I., Khartabil H., Duffey $R$. Heat transfer at supercritical pressures (survey)// Proc. 11-th Intern. Conf. on Nucl. Eng. Tokyo,Japan, April 20 - 23. -2003. Paper ICONE 11-36454.

5. Grabezhnaia V., Kirillov P. Heat transfer at supercritical pressures and boundaries of worsened heat // Teploenergetika. - 2006. - №4. P. 46 - 51. (Rus.)

6. Kirillov P, Lozhkin V., Smirnov A. The study boundaries degraded of worsened heat in channels with supercritical pressure // Report of IPPE-2988. - 2003. (Rus.)

7. Avramenko A.A., Basok B.I., Dmitrenko N.P., Kovetska M.M., Tyrinov A.I., Davydenko B.V. Renormalization group analysis of turbulence. K.: Expres.- 2013. - 299 p. (Rus.)

8. Alexandrov A., Orlov K., Ochkov $V$. Thermophysical properties of the working materials engineering.- M.: Pub. H. MPEI. - 2009. (Rus.)

9. Roach P. Computational fluid dynamics. M.: Mir. - 1980. -616 p. (Rus.)

10. Kurganov V., Maslakova I. Integral method for calculating heat transfer in pipes stabilized at about monophase near critical region // Thermophysic High temperatures. - 2010. - v. 48, № 4. - P. 568 - 581. (Rus.)

Получено 23.10.2014 Received 23.10.2014 